phys. stat. sol. (a) 190, No. 3, 625-629 (2002)

\title{
Level Repulsion of Localized Excitons in Disordered Quantum Wells
}

\author{
V. SAvona ${ }^{1}$ ) (a), E. Runge (a), R. Zimmermann (a), F. Intonti (b), \\ V. Emiliani (b), Ch. Lienau (b), and T. Elsaesser (b) \\ (a) Institut für Physik, Humboldt Universität, Hausvogteiplatz 5-7, 10117 Berlin, \\ Germany
}

(b) Max-Born-Institut für Nichtlineare Optik und Kurzzeitspektroskopie, Max-Born-Str. 2A, 12489 Berlin, Germany

(Received September 4, 2001; accepted September 10, 2001)

Subject classification: 07.79.Fc; 73.20.Fz; 78.55.Cr; 78.67.De; S7.12

The spectral autocorrelation for a set of over 400 near-field photoluminescence spectra of a narrow quantum well has been evaluated. It is compared with a microscopic model of an exciton in a disorder potential. The analysis provides strong evidence for quantum mechanical level repulsion and allows a quantitative estimate of the microscopic disorder features. The concept of participation ratio is invoked to quantify the exciton center-of-mass localization lengths. These turn out to be broadly distributed and increase rapidly with energy.

The connection between the spectral broadening of an exciton line in a quantum well (QW) and the presence of structural disorder has been known for two decades [1]. The center of mass $(\mathrm{COM})$ of the exciton is subject to energy fluctuations originating from interface and alloy disorder present in the heterostructure. Consequently, exciton states are randomly localized with an inhomogeneous distribution of eigenenergies. Within certain approximations, the inhomogeneous exciton can be mapped onto the more general problem of a quantum mechanical particle in a disordered potential [2]. Despite this connection and the accurate existing experimental characterizations [3-5], however, the interpretation of experiments is mostly of qualitative and empirical nature, often involving the concept of potential wells of typical lateral extension where the exciton COM gets trapped.

Recent spatially-resolved techniques [6], such as micro-PL and near-field scanning optical microscopy (NSOM) [7], have allowed to study the exciton spectrum on very small areas of the sample, with a size of the order of $200 \mathrm{~nm}$. They allow to resolve the sharp emission lines originating from the few localized excitons present in such small regions. By performing a statistical correlation analysis of many such spectra it should be possible, as we have suggested [8], to extract very precise quantitative information on the disordered system, namely the quantum mechanical level repulsion. Level repulsion arises from the quantum mechanical nature of the system which prevents spatially overlapping localized eigenstates of the system from being close in energy.

In this work, we combine state-of-the-art near-field spectral data obtained from a single QW with a detailed theoretical analysis based on the numerical solution of the Schrödinger equation for the exciton $\mathrm{COM}$ in a disordered, spatially correlated poten-

\footnotetext{
1) Corresponding author; e-mail: savona@physik.hu-berlin.de
} 
tial. The evaluation of the average autocorrelation function of spatially resolved spectra and the comparison with the corresponding simulated quantity provide the first quantitative evidence of level repulsion for a QW exciton. The theoretical model allows then to study the distribution of exciton sizes as a function of energy.

In our experiments, NSOM with a combined spatial and spectral resolution of $150 \mathrm{~nm}$ and $100 \mu \mathrm{eV}$ is used to obtain spatially resolved photoluminescence (PL) spectra. The excitation energy and excitation power coupled into the fiber probe had values of $1.96 \mathrm{eV}$ and $800 \mathrm{nW}$, respectively. As a model system, we investigated a $3 \mathrm{~nm}$ thick GaAs/ $\mathrm{Al}_{0.5} \mathrm{Ga}_{0.5} \mathrm{As}$ quantum well grown on a GaAs (311)A substrate [9] at a sample temperature of $20 \mathrm{~K}$. In Fig. 1 results from the PL experiments are summarized. The near-field PL spectra recorded with high spatial and spectral resolution are dominated
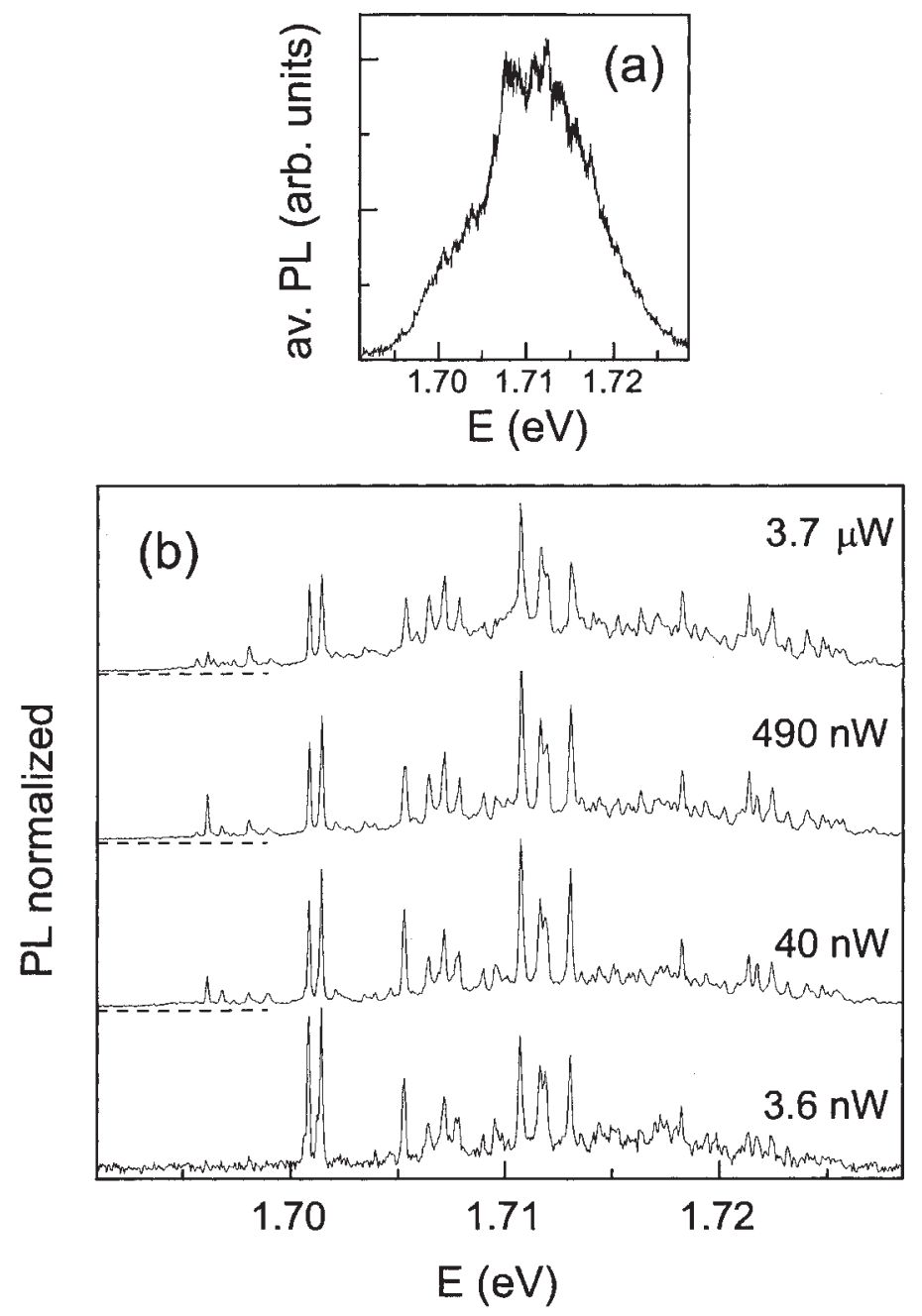

Fig. 1. a) Exciton PL spectrum obtained averaging the 432 near-field spectra. b) Typical near-field spectrum measured at various excitation intensities 
by a set of spectrally sharp and intense emission lines. In contrast, the spatially averaged PL spectrum is characterized by a $15 \mathrm{meV}$ broad structureless emission band (Fig. 1a). Panel b) shows one typical near-field spectrum measured for different excitation intensities coupled into the fiber. The spectral structures are practically unchanged within this intensity range. This rules out biexcitonic effects in the spectral autocorrelation.

A QW exciton, subject to in-plane energy fluctuations smaller than the exciton binding energy, can be described as a point particle moving in an effective potential with finite spatial correlation [2]. Thus, the eigenstates of the corresponding two-dimensional Schrödinger equation are localized. As a consequence of quantum mechanics, two spatially overlapping eigenstates are unlikely to have a very small energy separation, in the same way as for the well known avoided level crossing. On the contrary, states which lie spatially far apart are allowed to be quasi-degenerate. It is possible to quantify this feature by introducing the spectra autocorrelation function $R_{\mathrm{c}}(\Delta E)=R(\Delta E)-R_{0}(\Delta E)$, where

$$
\begin{aligned}
& R(\Delta E)=\left\langle\int \mathrm{d} E I_{n}(E) I_{n}(E-\Delta E)\right\rangle, \\
& R_{0}(\Delta E)=\int \mathrm{d} E\left\langle I_{n}(E)\right\rangle\left\langle I_{n}(E-\Delta E)\right\rangle .
\end{aligned}
$$

Here, the notation $\langle\cdots\rangle$ denotes the average over the measured spots in the experiment or over an ensemble of disorder realizations in the simulation. The quantity $I_{n}(E)$ represents the $n$-th optical spectrum of the ensemble under consideration. In our theory, we simulate the optical density spectrum, which under the present conditions is expected to provide the same information as the PL spectrum [10]. The quantity $R(\Delta E)$ represents the average distribution of energy-level distances weighted by the optical strengths, while $R_{0}(\Delta E)$ is its uncorrelated counterpart. By subtracting the two quantities, the contribution from pairs of uncorrelated levels cancels out and only those pairs of states whose relative distance is below their localization length contribute.

We would like to stress at this point that level repulsion does not imply the existence of a characteristic repulsion energy, as sometimes believed. This is true only in the strong localization case, in which most of the eigenstates are localized in a single valley of the potential. Assuming that the various potential minima have similar shape and size (e.g. in self-organized quantum dot samples), a typical energy separation is likely to arise in such a scenario and $R_{\mathrm{c}}(\Delta E)$ might be peaked at this energy value. However, if the system displays weak localization and the localized states span many potential fluctuations, the random shapes and positions of the states prevent the system from displaying a preferential energy splitting and level repulsion will result in a broad negative feature of $R_{\mathrm{c}}(\Delta E)$ at $\Delta E \rightarrow 0$ [11].

Our simulations are based on the one-particle Schrödinger equation with a disorder potential having an average amplitude $\sigma$ and a spatial correlation length $\xi$ [10]. The equation has been solved numerically over a square domain with $130 \mathrm{~nm}$ lateral size for many different realizations of the random potential. The autocorrelation function has been evaluated using Eqs. (1) and (2) and introducing a Lorentz broadening of the spectra which account for the homogeneous exciton linewidth and for the finite energy resolution of the experiment. The same equations have been used for the analysis of the experimental data. The parameters $\sigma$ and $\xi$ have been adjusted to fit the experimental autocorrelation curve, obtaining $\sigma=5.3 \mathrm{meV}$ and $\xi=17 \mathrm{~nm}$. The results are shown in Fig. 2. The agreement between theory and experiment is striking. The thin curve shows the theoretical result without introducing a Lorentz broadening. Here, the 


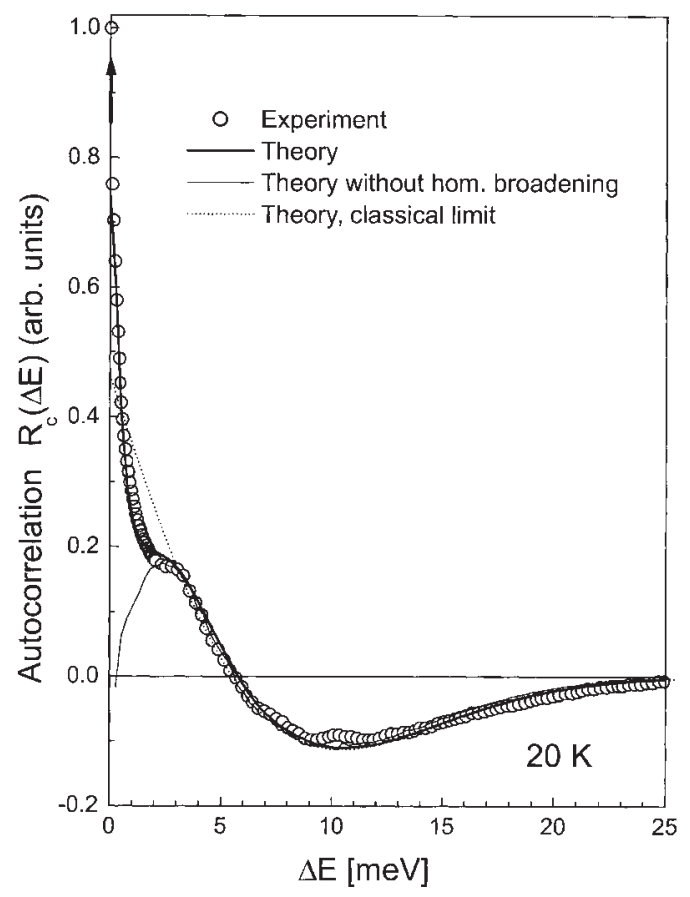

Fig. 2. Spectral autocorrelation function $R_{\mathrm{c}}(\Delta E)$. The arrow on the vertical axis denotes a Dirac delta function, which is part of the simulated autocorrelation without line broadening (thin line)

level repulsion appears as a dip for $\Delta E \rightarrow 0$. In addition, a delta-function is present denoting the contribution from the self-correlation of each exciton level. By introducing a Lorentz broadening, the delta function and the level repulsion dip are smeared out, and only a shoulder remains in the autocorrelation function. The broadening used for the theoretical curve is consistent with the linewidth of the spikes as measured in the individual near-field spectra. In absence of quantum mechanical level repulsion, we would expect a classical result in which $R_{\mathrm{c}}(\Delta E)$ is determined

by the correlations in the potential only. This result is shown in Fig. 2 as a dotted line for comparison. The marked difference with the experiment supports our interpretation in terms of quantum mechanical level repulsion.

The magnitude of the level repulsion dip is related to the spatial extension of the relevant COM wave functions. However, with the present model, and knowing the values of the parameters $\sigma$ and $\xi$, it is possible to extract more detailed wave function information. As an example, we evaluate the exciton localization length $\Lambda_{\alpha}$ via the participation ratio [12]

$$
\Lambda_{\alpha}^{-2}=\int \psi_{\alpha}^{4}(\mathbf{R}) \mathrm{d} \mathbf{R},
$$

where $\psi_{\alpha}(\mathbf{R})$ is the $\alpha$-th eigenstate of the exciton COM motion. Figure 3 shows the computed $\Lambda_{\alpha}$ as a function of the energy across the exciton spectrum. The dots are computed over 10 potential realizations, while the line is an optically weighted average. Two important features emerge from this plot. First, the exciton localization length is much larger than the potential correlation length $\xi=17 \mathrm{~nm}$, proving that the wave functions are localized over several minima of the disorder potential. In particular, this is inconsistent with an interpretation of level repulsion as being related to a single characteristic exciton size. Second, the localization length varies strongly as a function of energy and even for a given energy it is broadly distributed. This suggests that the idea of a unique exciton localization length for a given system, often claimed in the exciton literature, is highly inappropriate.

In conclusion, we have studied the autocorrelation function of many near-field exciton PL spectra obtained from a single QW by means of a microscopic theory of an exciton in a disordered QW. Our result is the first quantitative characterization of quan- 


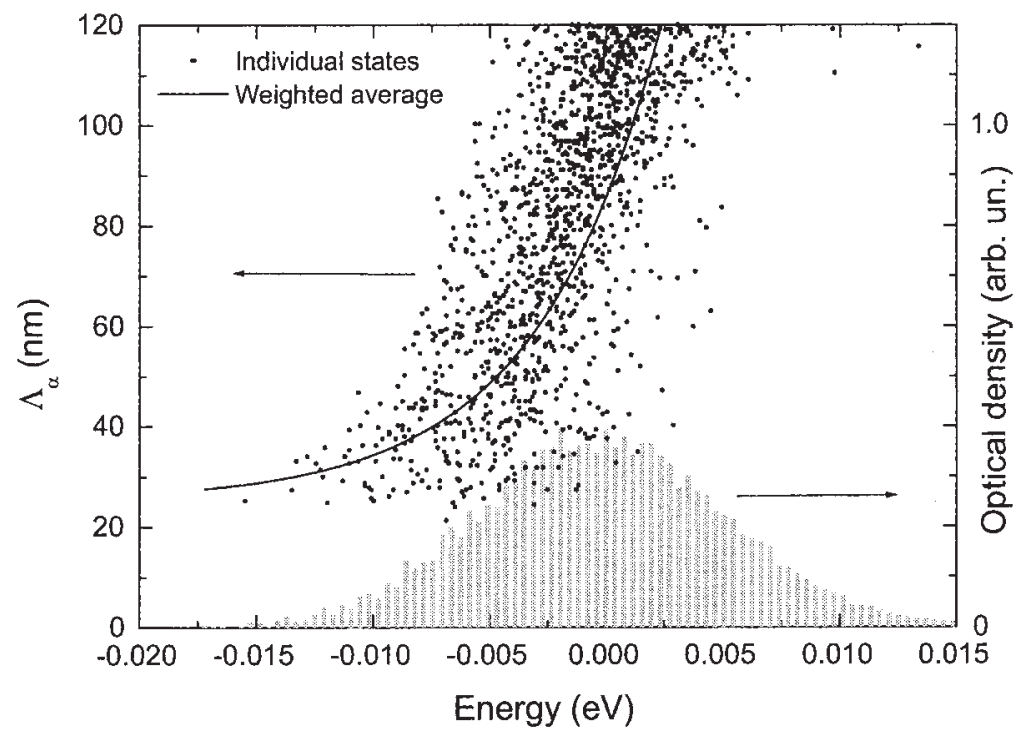

Fig. 3. Exciton localization length $\Lambda_{\alpha}$ (circles). The full line is the optically-weighted average of $\Lambda_{\alpha}$ as a function of energy. The histogram is the corresponding averaged exciton optical density as obtained from the simulation

tum-mechanical level repulsion. This phenomenon is generally predicted to occur in all low-dimensional disordered systems. Our analysis could therefore be the starting point for a systematic spectroscopic approach to the properties of disordered systems.

Acknowledgements V. S. and V. E. acknowledge the Marie Curie European Fellowships MCFI-1999-00728 and ERB40001GT975127 respectively.

\section{References}

[1] C. Weisbuch, R. Dingle, A. C. Gossard, and W. Wiegmann, Solid State Commun. 38, 709 (1981).

[2] R. Zimmermann, F. Grosse, and E. Runge, Pure Appl. Chem. 69, 1179 (1997).

[3] K. Brunner, G. Abstreiter, G. Böhm, G. Tränkle, and G. Weimann, Phys. Rev. Lett. 73, 1138 (1994).

[4] D. Gammon, E. S. Snow, B. V. Shanabrook, D. S. Katzer, and D. Park, Phys. Rev. Lett. 76, 3005 (1996).

[5] G. von Freymann, E. Kurtz, C. Klingshirn, and M. Wegenner, Appl. Phys. Lett. 77, 394 (2000).

[6] H. F. Hess, E. Betzig, T. D. Harris, L. N. Pfeiffer, and K. W. West, Science 264, 1740 (1994).

[7] G. Behme, A. Richter, M. Süptitz, and Ch. Lienau, Rev. Sci. Instrum. 68, 3458 (1997).

[8] E. Runge and R. Zimmermann, Adv. Solid State Phys. 38, 251 (1998).

[9] R. Nötzel, M. Ramsteiner, J. Menniger, A. Trampert, H.-P. Schönherr, L. Däweritz, and K. H. Ploog, Jpn. J. Appl. Phys. 35, L297 (1996).

[10] F. Intonti, V. Emiliani, C. Lienau, T. Elsaesser, V. Savona, E. Runge, R. Zimmermann, R. Nötzel, and K. H. Ploog, Phys. Rev. Lett. 87, 076801 (2001).

[11] V. Savona and R. Zimmermann, Phys. Rev. B 60, 4928 (1999).

[12] B. Kramer and A. MacKinnon, Rep. Prog. Phys. 56, 1469 (1993). 
